and national groups in the initial identification of the condition as well as challenges in management and logistics.

Mycyk further underscores the critical multiagency, multidisciplinary effort by groups such as the Illinois Poison Center, the Toxikon Consortium, the CDC, and the Illinois Department of Public Health. It was through the efforts of these organizations and the multidisciplinary collaborations of area hospital and emergency workers that the outbreak was recognized and effective treatment to achieve hemostasis and correct coagulopathy was initiated.

Wang et al. address pharmacoeconomic considerations in the use of vitamin $\mathrm{K}_{1}$. They describe the higher cost of the oral formulation than the intravenous formulation and raise concerns about procurement during shortages. As we reported, this became a major issue in central Illinois and led some patients to obtain vitamin $\mathrm{K}_{1}$ products from nonpharmaceutical online vendors. We recommended against this because of concerns about the purity of these supplements. Although local pharmacies were eventually able to acquire additional supplies from other states, a disseminated outbreak would require a coordi- nated effort to increase the availability of vitamin $K_{1}$. We strongly support exploration of alternative sources of vitamin $\mathrm{K}_{1}$ by the FDA in order to lower costs and circumvent future shortages.

Amar H. Kelkar, M.D.

University of Florida Shands Hospital

Gainesville, FL

amar.kelkar@medicine.ufl.edu

Michael D. Tarantino, M.D.

Jonathan C. Roberts, M.D.

Bleeding and Clotting Disorders Institute

Peoria, IL

Since publication of their article, the authors report no further potential conflict of interest.

1. Wroge L. Synthetic marijuana causes more cases of severe bleeding in Wisconsin. Madison: Wisconsin State Journal, September 19, 2018 (https://madison.com/wsj/news/local/crime/synthetic -marijuana-causes-more-cases-of-severe-bleeding-in-wisconsin/ article_47e0898f-1d49-530d-95d5-7ec7d3e21cbb.html).

2. Moritz E, Austin C, Wahl M, et al. Notes from the field: outbreak of severe illness linked to the vitamin $\mathrm{K}$ antagonist brodifacoum and use of synthetic cannabinoids - Illinois, March-April 2018. MMWR Morb Mortal Wkly Rep 2018;67:607-8. 3. Hussain N, Hussain F, Haque D, Saeed S, Jesudas R. An outbreak of brodifacoum coagulopathy due to synthetic marijuana in central Illinois. Mayo Clin Proc 2018;93:957-8.

4. Riley SB, Sochat M, Moser K, et al. Case of brodifacoumcontaminated synthetic cannabinoid. Clin Toxicol (Phila) 2018 August 27 (Epub ahead of print).

DOI: $10.1056 /$ NEJMc1814118

\title{
PCI Guided by Fractional Flow Reserve at 5 Years
}

TO THE EDITOR: Xaplanteris et al. (July 19 issue) ${ }^{1}$ compared outcomes of percutaneous coronary intervention (PCI) guided by measurement of the fractional flow reserve (FFR) with those of medical therapy in patients with stable coronary artery disease (Fractional Flow Reserve versus Angiography for Multivessel Evaluation [FAME] 2). The rate of spontaneous myocardial infarctions during the 5-year follow-up was higher in the medical-therapy group than in the PCI group (10.2\% vs. $6.5 \%$ ). In a parallel registry of patients without physiologically significant lesions who did not undergo randomization but received medical therapy, the rate was $7.8 \%$.

The authors state that their results "contradict the general belief that abrupt coronary occlusions occur predominantly at sites of mild stenosis and hence that the treatment of severe lesions may not prevent myocardial infarction." This affirmation does not appear to be justified by the data. The difference in the rate of myocardial infarctions between the two randomly assigned groups was not significant after adjustment for multiple comparisons. Importantly, there does not appear to have been any angiographic analysis of the site of acute occlusion. Myocardial infarctions could have occurred anywhere along the coronary vasculature, not necessarily on the substrate of the severe lesions that were not treated by PCI.

Peter Bogaty, M.D.

Quebec Heart and Lung Institute Quebec, QC, Canada peter.bogaty@fmed.ulaval.ca

No potential conflict of interest relevant to this letter was reported.

1. Xaplanteris P, Fournier S, Pijls NHJ, et al. Five-year outcomes with PCI guided by fractional flow reserve. N Engl J Med 2018; 379:250-9.

DOI: $10.1056 / N E J M c 1813100$ 
TO THE EDITOR: The FAME 2 trial $^{1}$ recommends measurement of FFR. However, according to the trial protocol, "In total occlusion or 'functional' total occlusion, the FFR measurements are not mandatory. In these lesions, the FFR value will arbitrarily set at 0.50 ." Recent revelations ${ }^{2}$ that the FFR was often inferred, rather than measured, undermine recommendations for FFR measurement.

The following questions arise. In how many patients in each group was the FFR inferred as 0.50 because of chronic total occlusion? How many patients per group had inferred FFRs of 0.50 because of "functional" chronic total occlusion? Functional chronic total occlusion sounds like very severe stenosis. However, the authors later revealed that these stenoses could be as mild as being approximately $40 \%$ in diameter ${ }^{2}$ and that the reason for the ascription of 0.50 was often not known. ${ }^{3}$ Would the authors please provide a histogram of the distribution of stenosis severity for the inferred 0.50 lesions in each study group, provide angiographic images of these lesions so clinicians can see when the FFR need not be measured, and show 5-year survival curves of patients in whom all FFRs were measured?

Darrel P. Francis, M.D.

Imperial College London

London, United Kingdom

darrel@drfrancis.org

Brahmajee K. Nallamothu, M.D.

University of Michigan

Ann Arbor, MI

Dr. Nallamothu reports being a co-inventor on a patent (US20170148158A1, "Automated Analysis of Vasculature in Coronary Angiograms") on software technology that uses signal processing and machine learning to automate the reading of coronary angiograms, held by the University of Michigan. No other potential conflict of interest relevant to this letter was reported.

1. De Bruyne B, Pijls NHJ, Kalesan B, et al. Fractional flow reserve-guided PCI versus medical therapy in stable coronary disease. N Engl J Med 2012;367:991-1001.

2. Ciccarelli G, Barbato E, Toth GG, et al. Angiography versus hemodynamics to predict the natural history of coronary stenoses: Fractional Flow Reserve Versus Angiography in Multivessel Evaluation 2 substudy. Circulation 2018;137:1475-85.

3. Barbato E, Toth GG, Johnson NP, et al. A prospective natural history study of coronary atherosclerosis using fractional flow reserve. J Am Coll Cardiol 2016;68:2247-55.

DOI: $10.1056 /$ NEJMc1813100

TO THE EDITOR: The 5-year results of the FAME 2 trial should prompt a renewed discussion regarding the appropriateness of contemporary clinical guidelines on intracoronary physiological variables for clinical decision making. The rate of major adverse cardiac events among patients with FFR-positive stenoses treated by medical therapy alone was only $27 \%$. This combined end point includes urgent revascularizations. Previous reports have questioned the appropriateness of using urgent revascularizations as an end point in an unblinded clinical trial.

Hence, a large majority (73\%) of patients in whom inducible ischemia is suspected according to the FFR, warranting revascularization according to contemporary guidelines, do not require PCI for prognostic purposes even in an unblinded study design biased against a medical-therapy approach. It can therefore be questioned whether current practice, in which all patients with FFRpositive lesions undergo PCI, is indeed appropriate, or whether we should put more effort into the development of diagnostic techniques that enhance the identification of patients who actually require coronary intervention for prognostic purposes. $^{1-4}$

Tim P. van de Hoef, M.D., Ph.D.

Jan J. Piek, M.D., Ph.D.

Amsterdam UMC

Amsterdam, the Netherlands

t.p.vandehoef@amc.uva.nl

Dr. van de Hoef reports receiving lecture fees from St. Jude Medical (now Abbott Vascular), Boston Scientific, and Volcano (now Philips Volcano) and travel fees from Acist Medical Systems. Dr. Piek reports receiving consulting fees from Abbott Vascular and Philips Volcano. No other potential conflict of interest relevant to this letter was reported.

1. van de Hoef TP, Siebes M, Spaan JA, Piek JJ. Fundamentals in clinical coronary physiology: why coronary flow is more important than coronary pressure. Eur Heart J 2015;36:3312-9a.

2. Gould KL, Johnson NP, Bateman TM, et al. Anatomic versus physiologic assessment of coronary artery disease: role of coronary flow reserve, fractional flow reserve, and positron emission tomography imaging in revascularization decision-making. J Am Coll Cardiol 2013;62:1639-53.

3. van de Hoef TP, van Lavieren MA, Damman P, et al. Physiological basis and long-term clinical outcome of discordance between fractional flow reserve and coronary flow velocity reserve in coronary stenoses of intermediate severity. Circ Cardiovasc Interv 2014;7:301-11.

4. van de Hoef TP, Echavarría-Pinto M, van Lavieren MA, et al. Diagnostic and prognostic implications of coronary flow capacity: a comprehensive cross-modality physiological concept in ischemic heart disease. JACC Cardiovasc Interv 2015;8:1670-80.

DOI: 10.1056/NEJMc1813100

TO THE EDITOR: At 5 years, the FAME 2 trial showed no significant benefit with respect to 
the rates of death or myocardial infarction among patients assigned to PCI who had hemodynamically significant lesions. The only significant difference between the PCI group and the medical-therapy group was in the rate of urgent revascularization. However, as the authors acknowledge, urgent revascularization was unblinded, making this end point subject to bias, as it was at the investigators' discretion whether to recommend PCI. There was no panel of independent experts or a priori objective criteria to guide this decision.

Furthermore, they state that, "Our results contradict the general belief that abrupt coronary occlusions occur predominantly at sites of mild stenosis and hence that the treatment of severe lesions may not prevent myocardial infarction." However, their data showed no significant between-group difference in myocardial infarction even though they were treating severe lesions (FFR, $\leq 0.80$ ). Finally, the benefit of PCI with respect to angina disappeared by 5 years of follow-up. Their data support the conclusion that PCI does not prolong life or prevent myocardial infarction even in patients with hemodynamically significant lesions, not that FFR-guided PCI in such patients is superior to medical therapy alone.

Dean Ornish, M.D.

Preventive Medicine Research Institute

Sausalito, CA

dean.ornish@pmri.org

Rita F. Redberg, M.D.

University of California, San Francisco

San Francisco, CA

Dr. Ornish reports writing books on health for which he receives royalties, receiving lecture honoraria from Harvard Medical School (for its annual Lifestyle Medicine CME course), the International Conference on Nutrition in Medicine, Cedars-Sinai Medical Center, Los Angeles (for medical grand rounds), the Leading Authorities speakers bureau for the Council for Responsible Nutrition, and the MDVIP national meeting, receiving consulting and licensing or royalty fees from Sharecare, serving on the advisory board of and receiving stock from Obvious Ventures, and receiving a salary from the Preventive Medicine Research Institute, a nonprofit 501(c)(3) public foundation that has received grants from the National Institutes of Health, Department of Defense, foundations (public and private), and individuals and that is currently conducting a randomized, controlled trial to determine whether comprehensive lifestyle changes may reverse the progression of early-stage Alzheimer's disease. No other potential conflict of interest relevant to this letter was reported.

DOI: $10.1056 /$ NEJMc1813100
THE AUTHORS REPLY: Bogaty correctly points out that a myocardial infarction could have occurred anywhere along the coronary vasculature. However, for each reported ischemic event, the FAME 2 investigators were requested to produce a narrative, including a description of the angiogram if available. On the basis of these narratives, we could identify the culprit lesion for 36 of the 53 myocardial infarctions reported as one of the components of the primary outcome in patients in the medical-therapy group of the FAME 2 trial, and we found that 32 of these lesions (89\%) were hemodynamically significant, with an FFR of 0.80 or less. Our data therefore do not support the contention that abrupt occlusions occur predominantly in mild stenoses.

As noted by Francis and Nallamothu, the FFR at baseline was inferred as 0.50 in 58 lesions with total occlusions and in 77 lesions for which the operator did not feel confident to advance a pressure wire. A total of 46 of these lesions were classified to have a stenosis with a $90 \%$ diameter, 23 to have a stenosis with a 70 to $90 \%$ diameter, and 8 to have a stenosis with a 50 to $69 \%$ diameter according to visual estimates. Only 28 randomly assigned patients were classified as having a hemodynamically significant lesion in the absence of at least one FFR measurement of 0.80 or less. Exclusion of these 28 patients did not change conclusions for the primary end point (hazard ratio, 0.45; 95\% confidence interval, 0.33 to $0.62 ; \mathrm{P}<0.001$ ) (Fig. 1). The protocol of the FAME 2 trial did not require central collection of baseline angiograms.

We agree with van de Hoef and Piek that not all FFR-positive lesions should undergo PCI. The decision to perform revascularization also depends on clinical factors, including age, symptoms, territory at risk, and the morphologic characteristics of the stenosis. The results of the FAME 2 trial indicate that on average patients benefit from PCI using second- or third-generation drug-eluting stents when at least one stenosis in a large epicardial artery is FFR-positive, but there seems to be a relationship between actual burden of disease characterized by FFR values at baseline and expected patient benefits. ${ }^{1}$ Additional metrics for the quantitative assessment of microvascular function are currently being developed and will probably refine the indications for revascularization. ${ }^{2}$ 
Stephane Fournier, M.D.

Lausanne University Center Hospital

Lausanne, Switzerland

Peter Jüni, M.D.

University of Toronto

Toronto, ON, Canada

Bernard De Bruyne, M.D.

Cardiovascular Center Aalst

Aalst, Belgium

bernard.de.bruyne@olvz-aalst.be

Since publication of their article, the authors report no further potential conflict of interest.

1. Barbato E, Toth GG, Johnson NP, et al. A prospective natural history study of coronary atherosclerosis using fractional flow reserve. J Am Coll Cardiol 2016;68:2247-55.

2. Xaplanteris P, Fournier S, Keulards DCJ, et al. Catheterbased measurements of absolute coronary blood flow and microvascular resistance: feasibility, safety, and reproducibility in humans. Circ Cardiovasc Interv 2018;11(3):e006194.

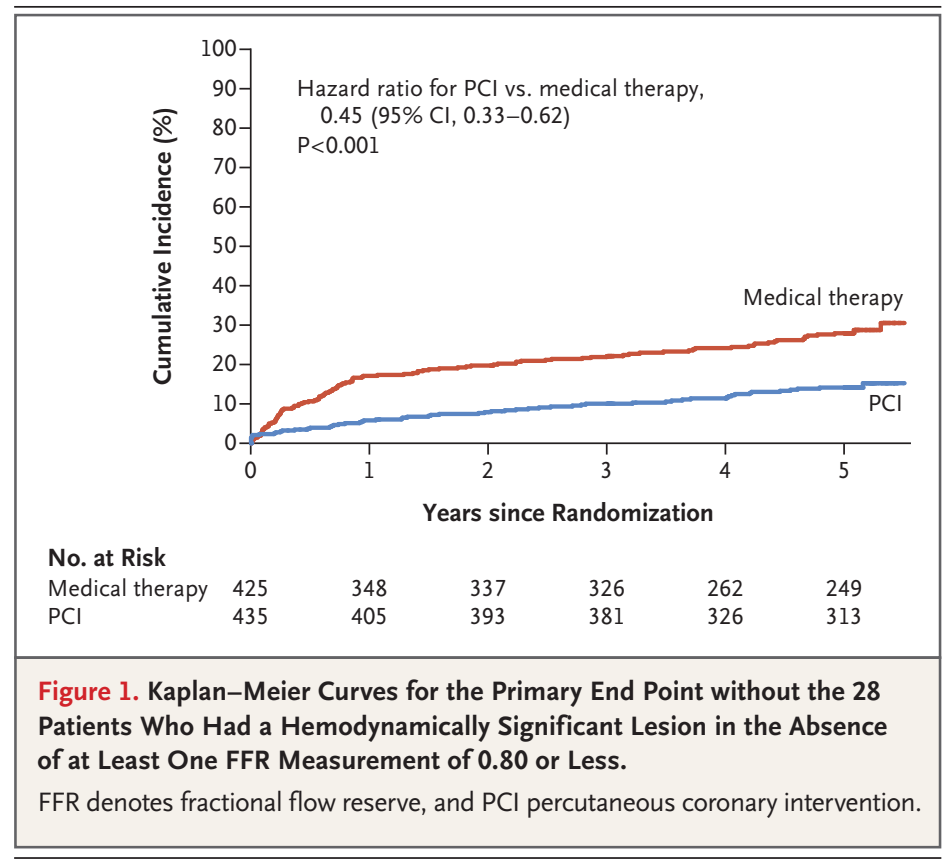

\section{Allogeneic BK Virus-Specific T Cells for PML}

TO THE EDITOR: Muftuoglu et al. (Oct. 11 issue $)^{1}$ describe the use of adoptive allogeneic BK virusspecific $\mathrm{T}$ cells for the treatment of progressive multifocal leukoencephalopathy (PML) and found that patients had alleviation of clinical symptoms and clearance of JC virus in the cerebrospinal fluid (CSF) after the infusion of donor-derived $\mathrm{T}$ cells. However, the infusion material could have included natural killer $\mathrm{T}$ cells, which are known to have antiviral activity. ${ }^{2}$

Furthermore, the patient with human immunodeficiency virus (HIV) infection could have recovered sufficient immunity from highly active antiretroviral therapy (HAART) to have adequate responses against JC virus and ameliorated PML symptoms. ${ }^{3}$ The infusion of largely CD4+ T cells, which entered the CSF and expressed CCR5, could, theoretically, create new HIV targets in the brain or traffic HIV-infected infused cells into the central nervous system (CNS), potentially resulting in HIV infection of the brain. Is it possible that the immune reconstitution syndrome that was seen in this patient could have been driven by the T-cell therapy itself $?^{4}$ This point is raised because in patients with lymphocytic cho- riomeningitis virus (LCMV) infection, LCMVspecific CD4+ cytolytic T cells in the CNS cause an immunopathological response. ${ }^{5}$

Daniela Marín-Hernández, M.D.

Luis P. Iñiguez, Ph.D.

Douglas F. Nixon, M.D., Ph.D.

Weill Cornell Medicine

New York, NY

dnixon@med.cornell.edu

No potential conflict of interest relevant to this letter was reported.

1. Muftuoglu M, Olson A, Marin D, et al. Allogeneic BK virusspecific $\mathrm{T}$ cells for progressive multifocal leukoencephalopathy. N Engl J Med 2018;379:1443-51.

2. Ohira $\mathrm{M}$, Ishiyama $\mathrm{K}$, Tanaka $\mathrm{Y}$, et al. Adoptive immunotherapy with liver allograft-derived lymphocytes induces antiHCV activity after liver transplantation in humans and humanized mice. J Clin Invest 2009;119:3226-35.

3. Roberts MT, Carmichael A, Lever AM. Prolonged survival in AIDS-related progressive multifocal leucoencephalopathy following anti-retroviral therapy and cidofovir. Int J Antimicrob Agents 2003;21:347-9.

4. Sainz-de-la-Maza S, Casado JL, Pérez-Elías MJ, et al. Incidence and prognosis of immune reconstitution inflammatory syndrome in HIV-associated progressive multifocal leucoencephalopathy. Eur J Neurol 2016;23:919-25.

5. Zajac AJ, Quinn DG, Cohen PL, Frelinger JA. Fas-dependent CD4+ cytotoxic T-cell-mediated pathogenesis during virus infection. Proc Natl Acad Sci U S A 1996;93:14730-5.

DOI: $10.1056 /$ NEJMc1815050 